\title{
Introduction of Multiscaled Longitudinal Vortices by Fractal-Patterned Surface Roughness
}

\author{
Manami Kosuda' ${ }^{1}$, Yoshihiro Kubota ${ }^{2}$, Masao Yokoyama ${ }^{3}$, Osamu Mochizuki ${ }^{4}$ \\ ${ }^{1}$ Graduate School of Science and Engineering, Toyo University, Saitama, Japan \\ ${ }^{2}$ Department of Mechanical Engineering, Toyo University, Saitama, Japan \\ ${ }^{3}$ Department of Information Science, Meisei University, Tokyo, Japan \\ ${ }^{4}$ Department of Biomedical Engineering, Toyo University, Saitama, Japan \\ Email: kubota548@toyo.jp
}

How to cite this paper: Kosuda, M., Kubota, Y., Yokoyama, M. and Mochizuki, O. (2019) Introduction of Multiscaled Longitudinal Vortices by Fractal-Patterned Surface Roughness. Journal of Flow Control, Measurement \& Visualization, 7, 120-132. https://doi.org/10.4236/jfcmv.2019.72010

Received: November 20, 2018

Accepted: April 25, 2019

Published: April 28, 2019

Copyright (c) 2019 by author(s) and Scientific Research Publishing Inc. This work is licensed under the Creative Commons Attribution International License (CC BY 4.0).

http://creativecommons.org/licenses/by/4.0/

\begin{abstract}
To determine the type of surface roughness pattern that is suitable for adaptive suppression of the drag of an obstacle, we observed flow structures introduced by such obstacles. Several roughness patterns were tested: geometric patterns, fractal patterns, reptile-skin patterns, and patterns of circular cylinders arranged in a lattice and in a zigzag manner. A suitable pattern for adaptive control of flow is one that generates longitudinal vortices with nonconstant distances. The preferred instability mode of a laminar boundary layer is expected to be selected automatically from fluctuations involving many frequencies and caused by fractal patterns. Snake- and reptile-skin patterns may have a similar ability as fractal patterns because they consist of multiscale patterns. The longitudinal vortices generated from peculiar positions and concave corners in patterns were observed. The distance between these vortices is not constant because the onset of vortices is at concave corners in fractal patterns. These vortices have differing strengths and easily cause nonlinear interactions, so they can disturb a laminar boundary layer with several higher-harmonic frequencies. The velocity profiles of the laminar boundary-layer flow over the fractal patterns were measured by using hydrogen bubbles. The results show the down-wash flow between the longitudinal vortices, which means that the vortices may effectively suppress the boundary layer separation in an adverse pressure gradient.
\end{abstract}

\section{Keywords}

Friction Drag Reduction, Surface Roughness, Fractal Pattern, Vortex, Flow Visualization 


\section{Introduction}

The aim of this study is to investigate how surface roughness patterns affect the fluid dynamic drag of moving obstacles. Friction drag due to the velocity gradient at the surface of an obstacle and the form drag related to the boundary layer separation due to the shape of an obstacle are the objects of this study. The reduction of friction drag in high-speed transport has been an object of fluid dynamics and involves the interaction between a boundary-layer flow and the obstacle surface. The friction factor of a pipe increases with increasing roughness relative to the diameter of the pipe. This friction factor may be determined from a Moody diagram. However, as the system reduces to the mini- and micro-scales [1] [2], the relative roughness exceeds $5 \%$, which is the limitation given by the Moody diagram. To obtain an accurate friction factor, Taylor et al. used a fractal [3] analysis to propose new roughness parameters (2006) [4] (the amplitude, spatial, hybrid, and functional parameters) to express the roughness.

If the design of the roughness pattern considers controlling flow structures such as a riblet [5] [6] [7], skin friction in the turbulent boundary layer may be reduced. Roughness patterns are disordered, whereas riblet patterns consist of parallel lines. How an arbitrarily shaped surface affects the turbulent skin friction was considered theoretically by Peet and Sagaut (2009) [5] based on the expression proposed by Fukagata et al. (2002) [8]. Friction-drag reduction was presented when the groove was optimized for laminar pressure-driven flows [7], which shows that it is possible to relate surface topology to flow dynamics.

Many surface patterns exist in nature; veins of leaves, turtle-shell blocks, fish or snake scales, butterfly scales, forked meandering streaming patterns on land, crack patterns in the dry ground, streak structures in a turbulent boundary-layer, etc. Wisdom for surviving environmental changes. Therefore, the relationship between patterns and skin friction should be studied (this constitutes a biomimetic approach). How the topical layer of birds [9], seals [10], other animals [11], sharks [12] [13] [14], and fish [15] [16] [17] [18] affects skin drag reduction has been studied by many researchers using techniques ranging from biomimetic to engineering. The design of micro surface patterns for surface coating is important for the fields of oleophobicity and philicity [19], water-repellency [20], hydrophobicity, lubrication, and tribology. A properly designed surface pattern can control turbulence [21] and reduce drag both passively [22] [23] and actively.

A passive way to reduce the drag involves introducing longitudinal vortices into a boundary layer by using vortex generators (VGs) [24]. AVG consists of pairs of small plates or short cylinders that generate pairs of clockwise and counterclockwise vortices. The vortex pairs induce a down-wash from the main stream to inside the boundary layer near the surface, as shown in Figure 1. If the VG is smaller than the turbulent-boundary-layer thickness, turbulence friction becomes small because it introduces order into the meandering of the turbulent streak structures. If the VG is larger than the turbulent-boundary-layer thickness, 


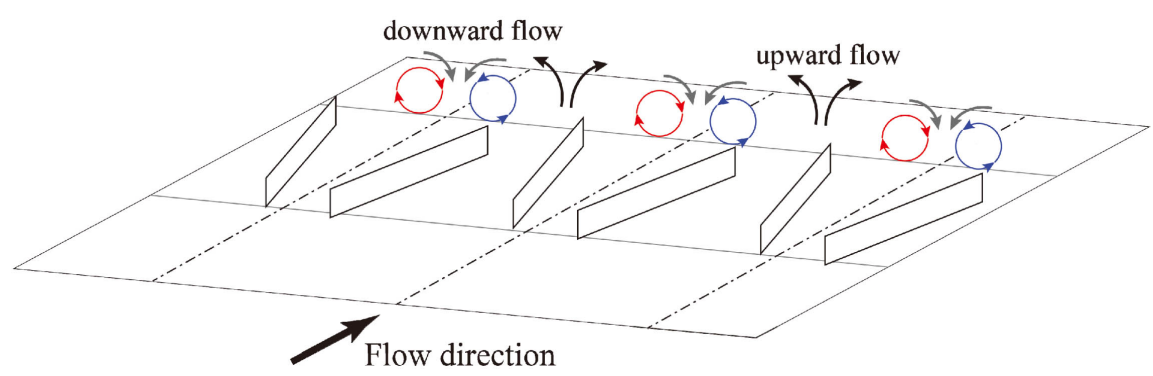

Figure 1. Sketch of flow induced by vortex generator.

the shape drag due to the separation becomes small because it suppresses the separation of the airplane wings and the cars. Godard et al. (2006) discuss how the optimized VG configuration reduces the frictional drag [25]. Their results show the difficulty of configuring VGs for a wide range of situations. By using VGs, we contribute to reducing the wall skin friction in a wide range of situations. The multi-scales of longitudinal vortices are formed in our study to interact with multi-scales of flow structure through the surface pattern of a flat plate. The change in drag of a circular cylinder with patterned roughness was investigated by Hojo (2015) [26] over a wide range around the critical Reynolds number. The drag of the circular cylinder is controllable through the combined arrangement and density of the lumped roughness pattern. The drag of the circular cylinder with many patterned textiles as a roughness was investigated by Hsu et al. (2018) [27]. The critical Reynolds number and pressure at $90^{\circ}$ measured from the front stagnation point are given by the empirical equation with the relative roughness height as a function.

To determine which type of surface roughness pattern is suitable for adaptively reducing the drag of an obstacle, we observed the flow structures introduced by the obstacle. Several roughness patterns were tested: geometric patterns, fractal patterns, reptile-skin patterns, and patterns with circular cylinders arranged in a lattice and in a zigzag manner. A suitable pattern for adaptive control of flow is one that generates longitudinal vortices with varying distances. The preferred instability mode of a laminar boundary layer is expected to be selected automatically from fluctuations involving many frequencies and given by fractal patterns. Fractal patterns are adopted to generate disturbances, including many wavelengths inspired from natural surface patterns because the local reduction of skin friction is expected from such natural patterns. The first step of this study is to determine which patterns facilitate the transition from a laminar to turbulent boundary layer and suppress separation. This study used flow-visualization experiments with dyes to investigate the tiny unevenness in surface patterns. Flow structures originating from the surface patterns were illuminated by a laser-light sheet, and were imaged by a CCD camera. The velocity profiles were then obtained from these movies. This paper discusses how fractal patterns affect the surface friction compared with the other patterns. 


\section{Experimental Setup and Surface Patterns}

A patterned plate was vertically immersed in an open water channel $0.18 \mathrm{~m}$ wide $\times 0.22 \mathrm{~m}$ high $\times 1.0 \mathrm{~m}$ long, as shown in Figure 2 . The free stream velocity was $0.05 \mathrm{~m} / \mathrm{s}$. The surface-patterns were put to the test plate, which was $5 \mathrm{~mm}$ thick, $150 \mathrm{~mm}$ high, and $100 \mathrm{~mm}$ long in the streamwise direction. The Reynolds number based on the free stream velocity and the plate length is $5.3 \times 10^{3}$. The boundary-layer flow is laminar.

Flow visualization experiments using the dye method were carried out to investigate the tiny unevenness of the surface patterns. The laser-light sheet illuminated a parallel plane close to the surface pattern to visualize the fine structure that originates from the surface patterns, as shown in Figure 2. The laser-light sheet settled just above the surface structure of the plate. The flow structures were colored by Rhodamine B, which fluoresces when illuminated in the orange, and imaged by a CCD camera. The laser wavelength was $532 \mathrm{~nm}$.

Several roughness patterns were tested: geometric patterns, fractal patterns, a snake-skin pattern, and groups of circular cylinders placed on lattice points or in the hound's tooth pattern. The preferred instability mode of a laminar boundary layer is expected to be selected automatically from fluctuations involving many frequencies given by the fractal patterns. Both snake-and lizard-skin patterns may have capacities similar to fractal patterns because they consist of multi-scaled patterns. The surface patterns tested are shown in Figure 3 and Figure 4. The groups of circular cylinders placed on lattice points (Figure 3(a)) or in a hound's tooth pattern (Figure 3(b)) were tested as representative geometric patterns. The knit pattern (Figure $3(\mathrm{c})$ ) was tested as an artificial pattern. The Sierpinski-gasket pattern (Figure 3(d)), Koch-curve pattern (Figure 3(e)), and multi-scale hexagon (Figure 3(f)) were adopted as fractal patterns. The characteristic width of the Sierpinski-gasket pattern (Figure 3(d)), Koch-curve pattern (Figure 3(e)), and multi-scale hexagon (Figure 3(f)) were the same. Figure 4(a) and Figure 4(b) show the side leather of a Python's belly and of a lizard's back, respectively. The red lines in Figure 4 show the location of the initial dye.

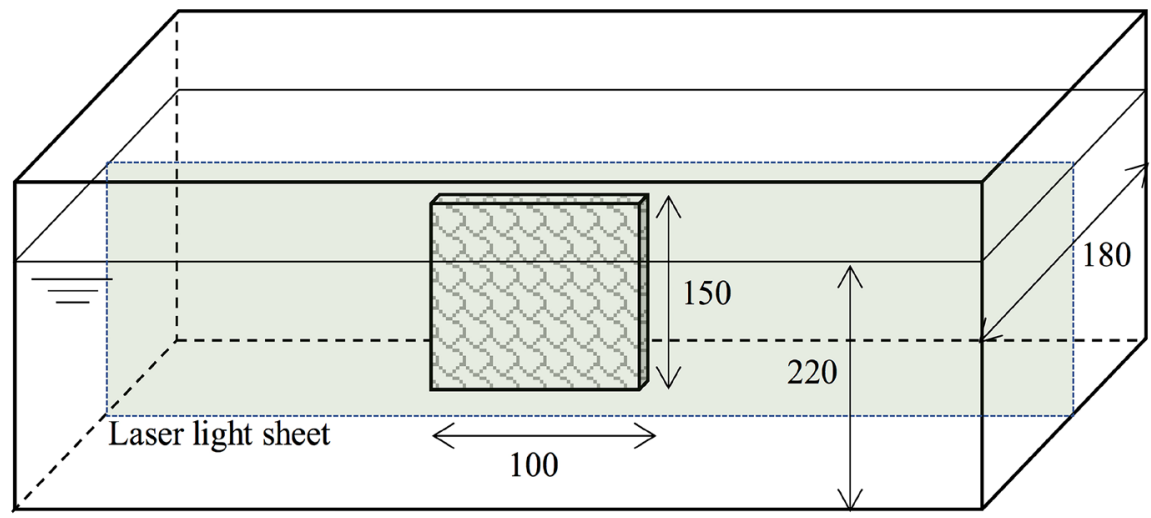

Figure 2. Test plate is set up in open channel, and a laser-light sheet illuminates a plane close to the plate. Unit in $\mathrm{mm}$. 


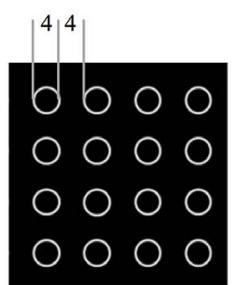

(a)

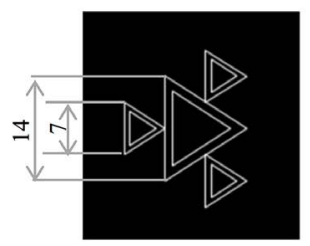

(d)

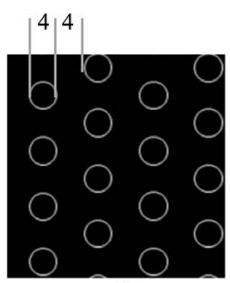

(b)

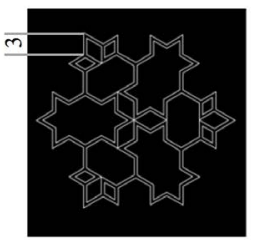

(e)

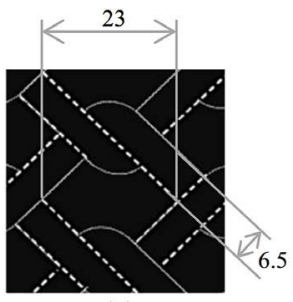

(c)

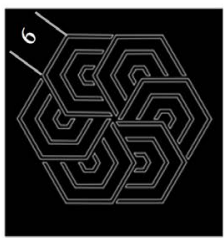

(f)

Figure 3. (a)-(c) Geometric patterns and (d)-(f) fractal patterns. Unit in mm.
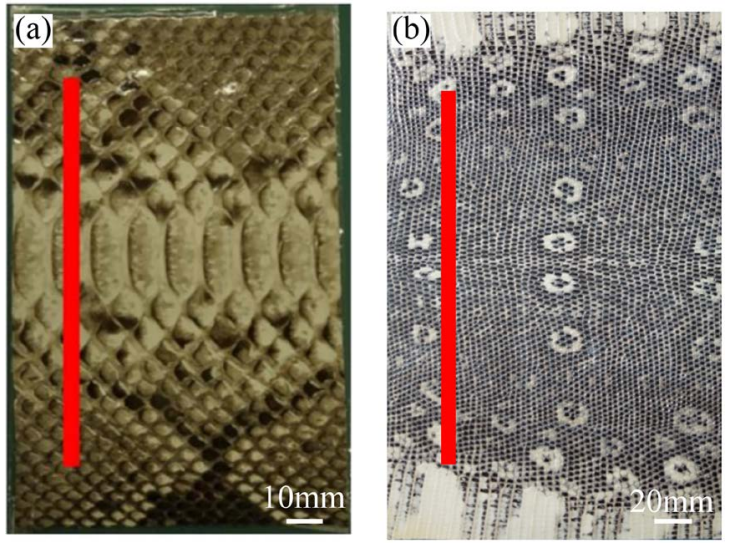

Figure 4. (a) Python-belly side leather; and (b) lizard-back side leather.

Velocity profiles of the boundary layer over the patterned roughness were obtained by using hydrogen-bubble lines. Hydrogen bubbles were produced from a positive-DC-biased tungsten wire. The diameter of the wire was $0.05 \mathrm{~mm}$ it was placed in the water tank $30 \mathrm{~mm}$ above the plate. A copper plate connected to a negative electrode was placed downstream of the water tank. A digital camera was used to record the flow patterns by means of hydrogen bubbles. The camera resolution was $1920 \times 1080$ pixels.

\section{Results}

\subsection{Geometric Pattern}

In Figure 5, the main stream flows from left to right, which shows the flow structures that are generated by each circular cylinder in the group. The visualization was done multiple times to confirm the repeatability and stability of the flow. The results are consistent between trials. Starting from the second row, longitudinal vortices are bundled between circular cylinders (see Figure 5(a)). The formation of longitudinal vortices was confirmed by the visualization of a 

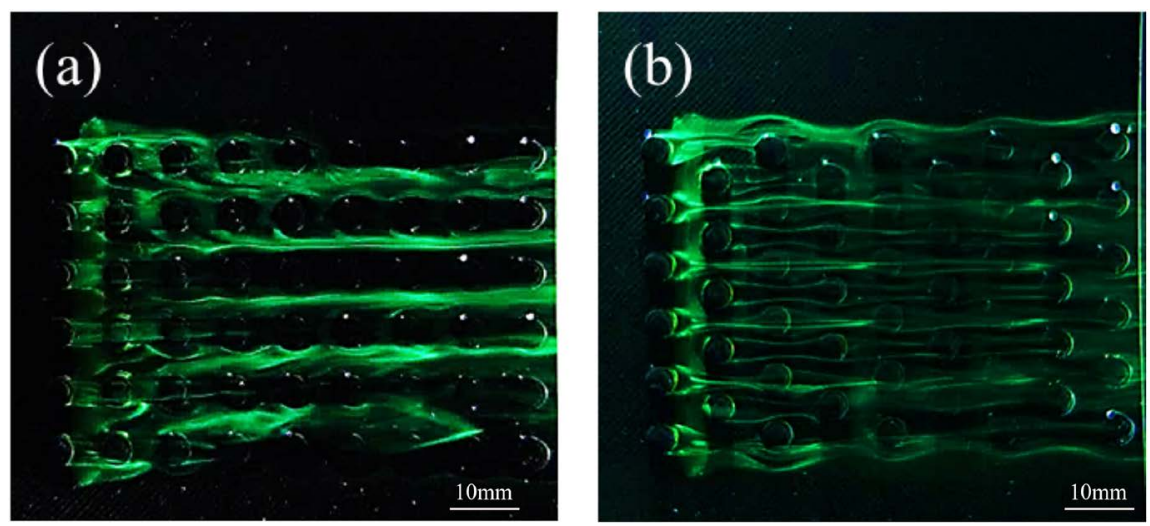

Figure 5. Longitudinal vortices of a group of circular cylinders placed on (a) a lattice or (b) on a hound's tooth pattern.

cross section of the illuminated region. This introduces strong longitudinal vortices into the boundary layer. For the hound's tooth pattern, the longitudinal vortices from the first row collide with the cylinders in the third row. In the same manner, the longitudinal vortices from the second row collide with the cylinders in the fourth row, and so on. The feedback mechanism between the separation points of the cylinder in the first row and stagnation point of the cylinder in the third row produces alateral disturbance to the bundled longitudinal vortices (see Figure 5(b)). Thus, the wavelength of the bundled longitudinal vortices shown in Figure 5(b) includes a fluctuation whose wavelength is twice as long as in Figure 5(a). The flow patterns around four cylinders in a square configuration were presented by Lam et al. (2003) [28]. This shows that strong fluctuating forces and serious vibration are introduced into the flow when the resonance occurs due to the wake impinging on the downstream cylinder.

For the knit pattern, clear vortices do not appear, but the dye accumulates at points indicated by the red circles in Figure 6, which are the intersections of grooves. These points may produce velocity fluctuations at some wavelengths because of the distance of these stagnations. Some groove intersections are initially marked by a spot of dye. This figure shows how the height of the bumpy pattern affects the change in velocity. The top, middle, and bottom figures show the flow over a 0.3-, 0.6-, and 0.9-mm-high pattern. The relatively clear effect can be seen in the bottom panel, even if it is recognized in the top panel. The flow pattern indicates weak longitudinal vortex structures, which are characterized by the knit pattern. The velocity profile at the center of a vortex pair must have an inflection point because of upward flow. Thus, the velocity gradient at the wall becomes small compared with the other location, which means that the shear stress is locally depressed at the position. This can be explained by invoking the effect of the vortex generator.

\subsection{Fractal Patterns}

Figures 7(a)-(c) show the longitudinal vortices generated by fractal patterns: the Sierpinski-gasket pattern (Figure 7(a)), Koch-curve pattern (Figure 7(b)), and 

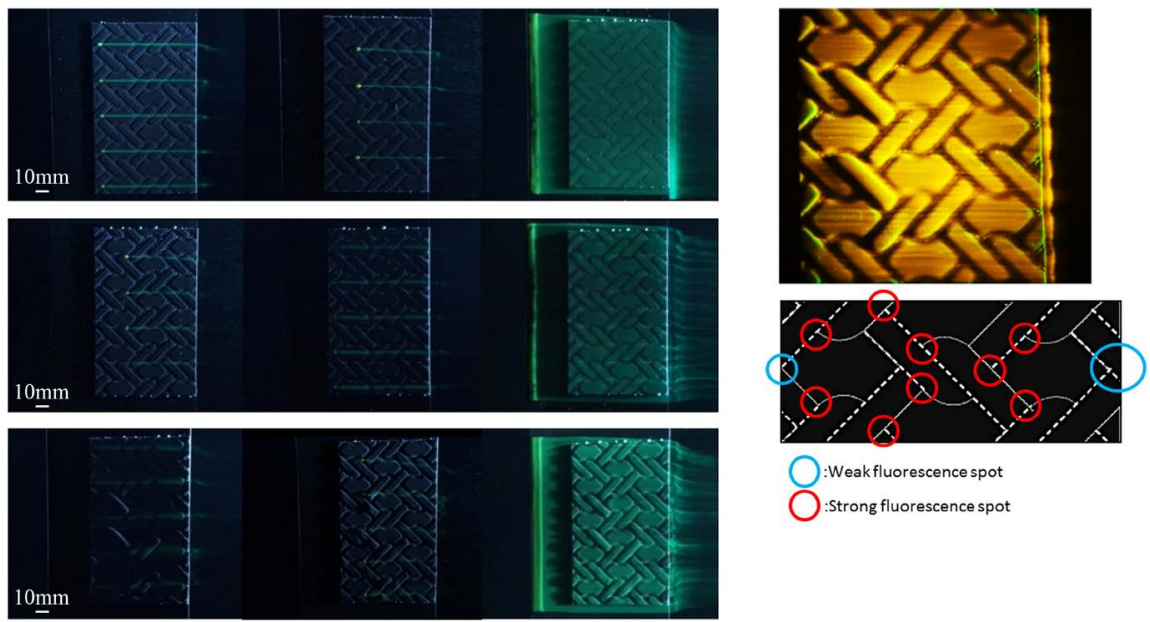

Figure 6. Stagnations in knit patterns. Bump is (top) $0.3 \mathrm{~mm}$ high, (middle) $0.6 \mathrm{~mm}$ high, and (bottom) $0.9 \mathrm{~mm}$ high. Each photo in top, middle, and bottom panels is of different marked position.
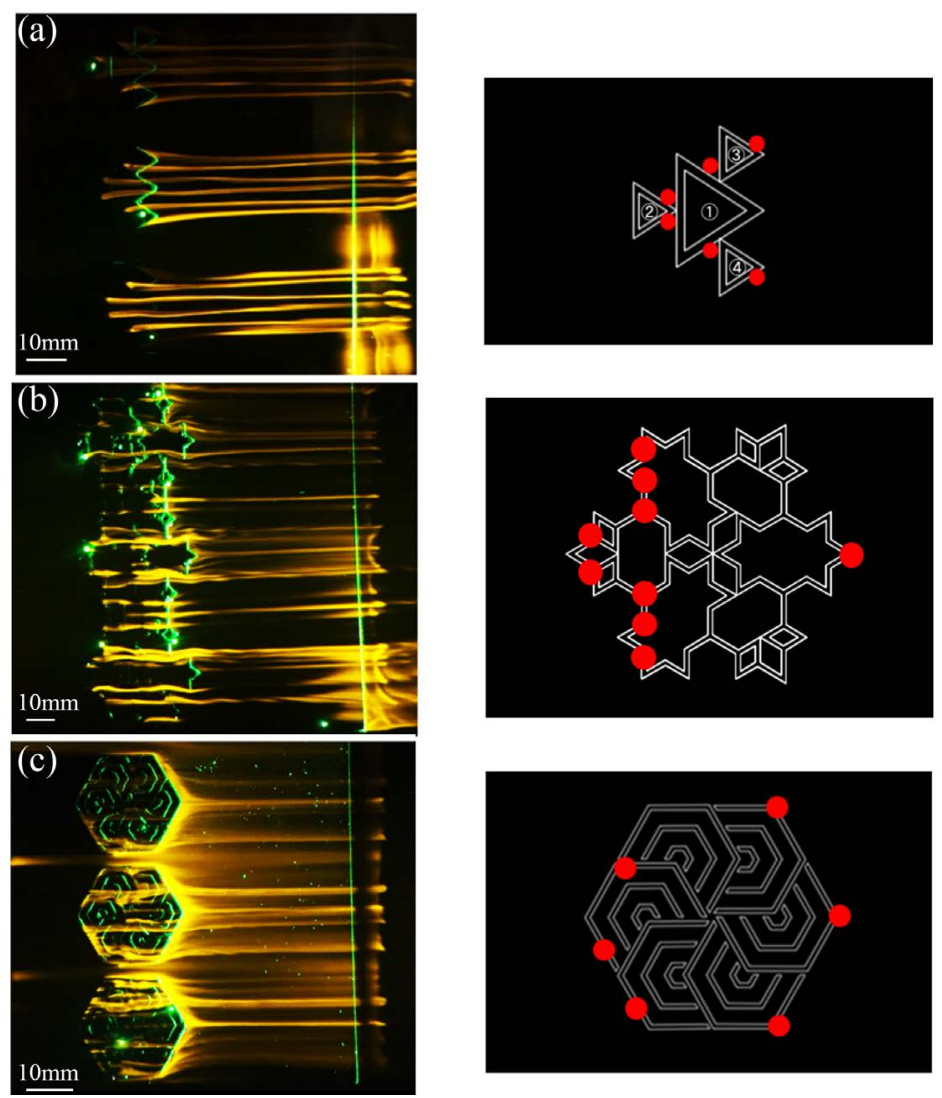

Figure 7. (a) Sierpinski-gasket pattern; (b) Koch-curve pattern; and (c) multiscale hexagon.

multiscale hexagon (Figure 7(c)). The red points in Figures 5(d)-(f) show the origin of the longitudinal vortices. The clear pairs of the longitudinal vortices which rotate each in different directions are recognized for the Sierpinski-gasket pattern. This pattern can be used to suppress a separation of a shear layer be- 
cause the vortex pair can introduce energy from the main stream into the boundary layer.

The fine flow structures seen in the Koch-curve pattern (Figure 7(b)) show complex patterns. The longitudinal vortices generated at the front convex points interact with rear intersections and corners of the pattern. Thus, some are enhanced but some are weakened by the interaction. Velocity fluctuations given by this pattern may include a change in velocity with different frequencies. The longitudinal vortices are wavy, so the vortices may include lateral fluctuations.

The distribution of the longitudinal vortices seen in the multiscale hexagon is biased; however, this seems not to be a clear rule of the starting point of longitudinal vortices, which means that the hexagon pattern can introduce unexpected disturbances into a flow.

\subsection{Reptile-Skin Patterns}

Patterns of fluorescein rhodamine dye flow near the surface are visualized by illumination with a black light. The design of the surface pattern visualized in this experiment is based on the scale pattern from the side of a python's belly. Some grooveinter sections are initially marked by a spot of rhodamine dye. Figure 8 shows how the incline angle of the pattern with respect to the main stream affects the fine structures generated by the snake-skin pattern. The shape and size of the scales consisting of the pattern differ at each place. The large scales elongated in the lateral direction are aligned in the longitudinal direction along the center line in the belly side. Small rhombus-shaped scales are aligned beside large scales.

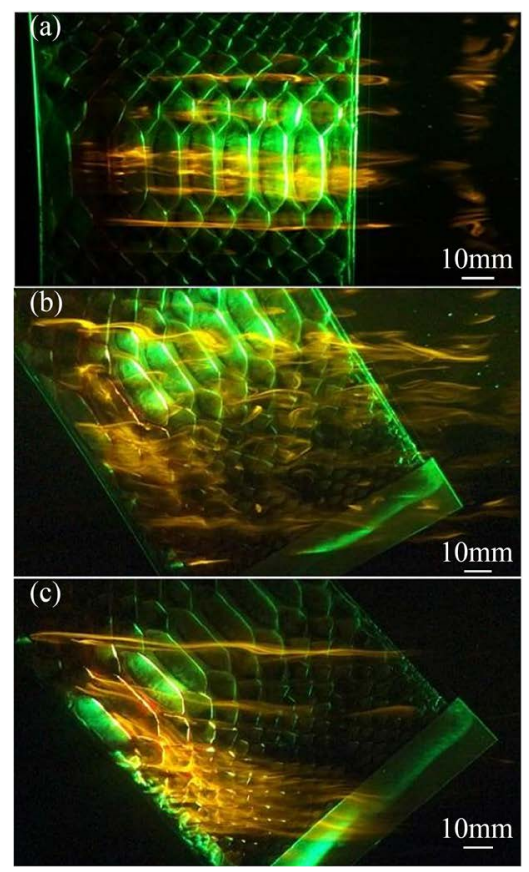

Figure 8. Flow structures over side leather of python's belly. Incline angle with respect to the main stream is (a) $0^{\circ}$; (b) $30^{\circ}$; and (c) $45^{\circ}$. 
Because snakes wiggle, we tested the effect of the inline angles with respect to the main stream. The fine structure generated by the snake skin is shown in Figure 8 (b) and Figure 8 (c) for different inline angles. For $0^{\circ}$, the shear flow in the boundary layer undergoes wavy fluctuations perpendicular to the surface when they pass over the large scales. Longitudinal vortices arise at a tip of rhombus-shaped scales. For $30^{\circ}$, the wavy longitudinal vortices appear clearly. The wavelength seems to be characterized by the distance between adjacent scales. For $45^{\circ}$, the longitudinal vortices are less clear compared with the inline angles.

Figure 9 shows the flow structures on the side leather of a lizard's back. Straight longitudinal vortices are lined up side by side interval the same size as the projections, which is a remarkable peculiarity. Pairs of large, three-dimensional vortex structures appear near the edge of the various patterns.

\subsection{Velocity Profiles}

The velocity profiles of a boundary layer flow passing over the surface patterns are visualized by using hydrogen bubbles. Figure 10 shows an example of visualized velocity profiles generated every $0.5 \mathrm{~s}$ over the center of a Sierpinski-gasket pattern. The averaged velocity profile was obtained from the photos. The results are shown in Figure 11 for flows over several roughness patterns. The uncertainty of measurement determined from the flow speed is $\pm 5 \%$. This is shown as error bar in a figure. The result with the smooth surface is shown together for the comparison. It is clear that all velocity profiles are pushed down compared with the velocity profile over a smooth surface, which means that the longitudinal vortices transfer energy from the main stream into the boundary layer. The most energy-efficient surface pattern is the Sierpinski gasket, as seen in Figure 7 (a), because it generates strong pairs of the longitudinal vortices.

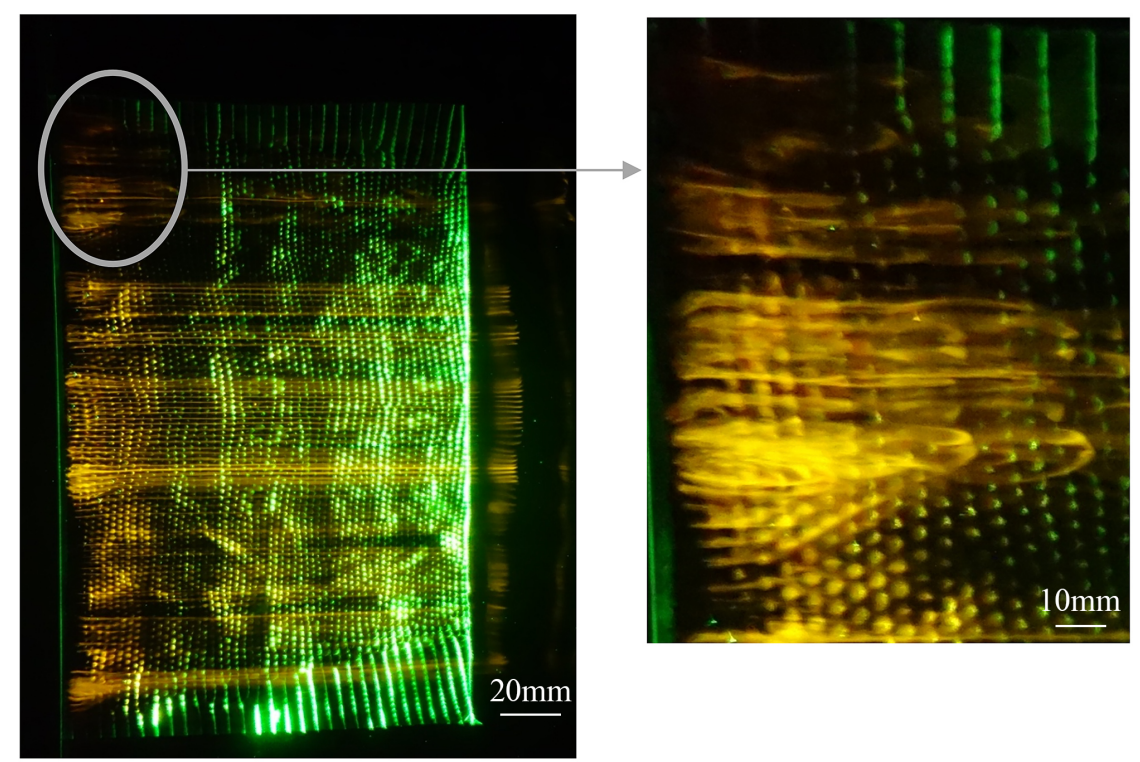

Figure 9. Flow structures on side leather of a lizard's back. 


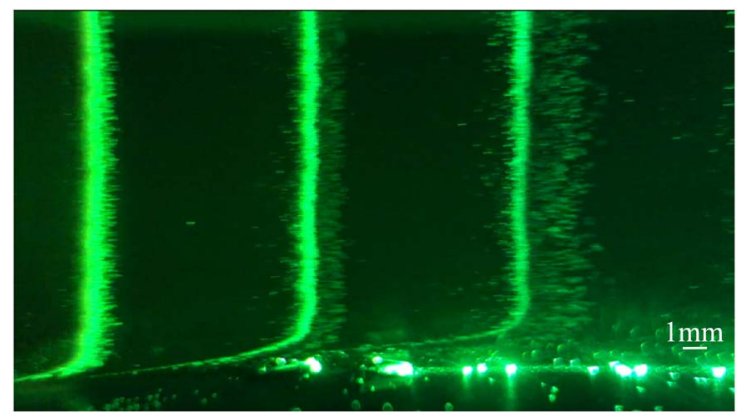

Figure 10. Example of visualized velocity profile of laminar boundary layer over center of a Sierpinski-gasket pattern.

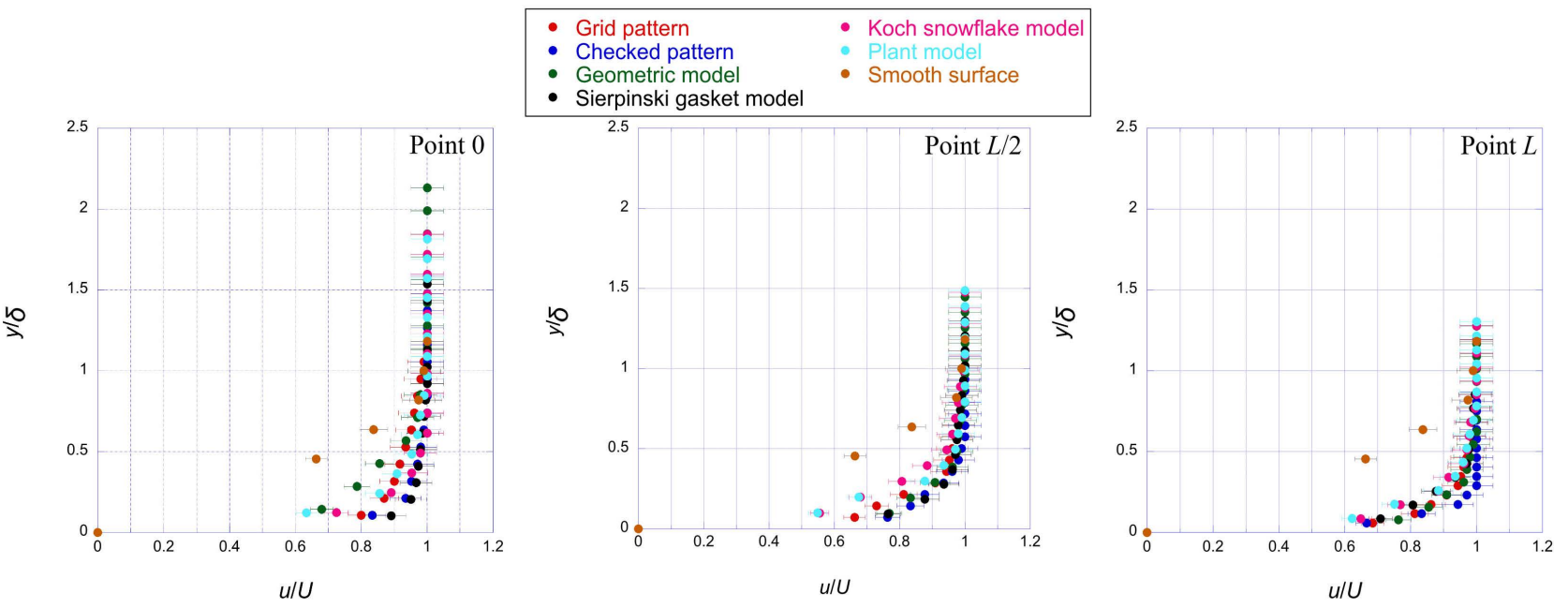

Figure 11. Comparison of velocity profiles of boundary-layer flow passing over surface patterns.

\section{Conclusion}

Surface patterns based on fractal patterns may suppress separation and reduce skin friction drag. This relates the change in a local velocity profile due to the longitudinal vortices generated by fractal surface patterns. The longitudinal vortices include the multiscale wavelength because of the self-similarity of the fractal patterns. These produce multi-frequency fluctuations in the laminar boundary layer. Because the boundary-layer flow uses the most favorable frequency, fractal patterns expedite the transition from a laminar to a turbulent boundary layer. The roughness pattern of the plate affects the main-flow structure by a multiscale of longitudinal vortices as optimized VGs. The Sierpinski gasket pattern introduces a strong pair of the longitudinal vortices.

\section{Conflicts of Interest}

The authors declare no conflicts of interest regarding the publication of this paper.

\section{References}

[1] Ahmmed, K.M.T. and Kietzig, A.M. (2016) Drag Reduction on Laser-Patterned 
Hierarchical Superhydrophobic Surfaces. Soft Matter, 12, 4912-4922. https://doi.org/10.1039/C6SM00436A

[2] Geraldi, N.R., Dodd, J.E., Xu, B.B., Wells, G.G., Wood, D., Newton, M. and McHale, G. (2017) Drag Reduction Properties of Superhydrophobic Mesh Pipes. Surface Topography: Metrology and Properties, 5, Article ID: 034001. https://doi.org/10.1088/2051-672X/aa793b

[3] Cottin-Bizonne, C., Barentin, C. and Bocquet, L. (2012) Scaling Laws for Slippage on Superhydrophobic Fractal Surface. Physics of Fluids, 24, Article ID: 012001. https://doi.org/10.1063/1.3674300

[4] Taylor, J.B., Carrano, A.L. and Kandlikar, G. (2006) Characterization of the Effect of Surface Roughness and Texture on Fluid Flow-Past, Present, and Future. International Journal of Thermal Sciences, 45, 962-968. https://doi.org/10.1016/j.ijthermalsci.2006.01.004

[5] Peet, Y. and Sagaut, P. (2009) Theoretical Prediction of Turbulent Skin Friction on Geometrically Complex Surfaces. Physics of Fluids, 21, Article ID: 105105. https://doi.org/10.1063/1.3241993

[6] Garcia-Mayoral, R. and Jiménez, J. (2011) Drag Reduction by Riblets. Philosophical Transactions of the Royal Society of London A, 369, 1412-1427. https://doi.org/10.1098/rsta.2010.0359

[7] Mohammadi, A. and Floryan, J.M. (2013) Groove Optimization for Drag Reduction. Physics of Fluids, 25, Article ID: 113601. https://doi.org/10.1063/1.4826983

[8] Fukagata, K., Iwamoto, K. and Kasagi, N. (2002) Contribution of Reynolds Stress Distribution to the Skin Friction in Wall-Bounded Flows. Physics of Fluids, 14, 73-76. https://doi.org/10.1063/1.1516779

[9] Pennycuick, C.J., Holliday, H., Obrecht III and Fuller, M.R. (1988) Empirical Estimates of Body Drag of Large Waterfowl and Raptors. Journal of Experimental Biology, 135, 253-264.

[10] Ito, M., Tamano, S., Iguchi, R., Yokota, K., Akino, N., Hino, R. and Kubo, S. (2006) Turbulent Drag Reduction by the Seal Fur Surface. Physics of Fluids, 18, Article ID: 065102. https://doi.org/10.1063/1.2204849

[11] Favier, J., Dauptain, A., Basso, D. and Bottaro, A. (2009) Passive Separation Control Using a Self-Adaptive Hairy Coating. Journal of Fluid Mechanics, 627, 451-483. https://doi.org/10.1017/S0022112009006119

[12] Wen, L., Weaver, J.C. and Lauder, G.V. (2014) Biomimetic Shark Skin: Design, Fabrication and Hydrodynamic Function. Journal of Experimental Biology, 217, 1656-1666. https://doi.org/10.1242/jeb.097097

[13] Pu, X., Li, G. and Liu, Y. (2016) Progress and Perspective of Studies on Biomimetic Shark Skin Drag Reduction. ChemBioEng Reviews, 3, 26-40.

https://doi.org/10.1002/cben.201500011

[14] Dean, B. and Bhushan, B. (2018) Shark-Skin Surface for Fluid-Drag Reduction in Turbulent Flow: A Review. Philosophical Transactions of the Royal Society A, 368, 4775-4806. https://doi.org/10.1098/rsta.2010.0201

[15] Lauder, G.V., Wainwright, D.K., Domel, A.G., Weaver, J.C., Wen, L. and Bertoldi, K. (2016) Structure, Biomimetics, and Fluid Dynamics of Fish Skin Surfaces. Physical Review Fluids, 1, Article ID: 060502. https://doi.org/10.1103/PhysRevFluids.1.060502

[16] Fletcher, T., Altringham, J., Peakall, J., Wignall, P. and Dorrell, R. (2016) Hydrodynamics of Fossil Fishes. Proceedings of the Royal Society B, 281, Article ID: 
20140703.

[17] Wu, L., Jiao, Z., Song, Y., Liu, C., Wang, H. and Yan, Y. (2018) Experimental Investigations on Drag-Reduction Characteristics of Bionic Surface with Water-Trapping Microstructures of Fish Scales. Scientific Reports, 8, Article No. 12186. https://doi.org/10.1038/s41598-018-30490-x

[18] Palmer, C. and Young, M.T. (2018) Surface Drag Reduction and Flow Separation Control in Pelagic Vertebrates, with Implications for Interpreting Scale Morphologies in Fossil Taxa. Royal Society Open Science, 2, Article ID: 140163. https://doi.org/10.1098/rsos.140163

[19] Bhushan, B. (2011) Biomimetics Inspired Surfaces for Drag Reduction and Oleophobicity/Philicity. Beilstein Journal of Nanotechnology, 2, 66-84.

https://doi.org/10.3762/bjnano.2.9

[20] Evans, H.B., Hamed, A.M., Gorumlu, S., Doosttalab, A., Aksak, B., Chamorro, L.P. and Castillo, L. (2017) Engineered Bio-Inspired Coating for Passive Flow Control. Proceedings of the National Academy of Sciences of the United States, 115, 1210-1214. https://doi.org/10.1073/pnas.1715567115

[21] Siva, M., Murugan, M., Sivasathya, U. and Dharmalingam, S. (2015) Biomimetic in Turbulence Reduction-Recent Developments. The Journal of Applied Sciences Research, 11, 123-134.

[22] Song, K.S., Kang, S.O., Jun, S.O., Park, H.I., Kee, J.D., Kim, K.H. and Lee, D.H. (2012) Aerodynamic Design Optimization of Rear Body Shapes of a Sedan for Drag Reduction. International Journal of Automotive Technology, 13, 905-914. https://doi.org/10.1007/s12239-012-0091-7

[23] Gu, Y., Liu, T., Mu, J., Shi, Z. and Zhou, P. (2017) Analysis of Drag Reduction Methods and Mechanisms of Turbulent. Applied Bionics and Biomechanics, 2017, Article ID: 6858720. https://doi.org/10.1155/2017/6858720

[24] Pujals, G., Depardon, S. and Cossu, C. (2010) Drag Reduction of a 3D Bluff Body Using Coherent Streamwise Streaks. Experiments in Fluids, 49, 1085-1094. https://doi.org/10.1007/s00348-010-0857-5

[25] Godard, G. and Stanislas, M. (2006) Control of a Decelerating Boundary Layer. Part 1: Optimization of Passive Vortex Generators. Aerospace Science and Technology, 10, 181-191. https://doi.org/10.1016/j.ast.2005.11.007

[26] Hojo, T. (2015) Control of Flow around a Circular Cylinder Using a Patterned Surface. WIT Transaction on Modeling and Simulation, 59, 245-256. https://doi.org/10.2495/CMEM150221

[27] Hsu, X.Y., Miau, J.J., Tsai, J.H., Lai, Y.H., Ciou, Y.S., Shen, P.T., Chuang, P.C. and Wu, C.M. (2018) The Aerodynamic Roughness of Textile Materials. Journal of the Textile Institute, 110, 771-779.

[28] Lam, K., Li, J.Y., Chan, K.T. and So, R.M.C. (2003) Flow Pattern and Velocity Field Distribution of Cross-Flow around Four Cylinders in a Square Configuration at a Low Reynolds Number. Journal of Fluids and Structure, 17, 665-679. https://doi.org/10.1016/S0889-9746(03)00005-7 


\section{Nomenclature}

$u$ : stream velocity.

$U$ : main stream velocity.

$y$. vertical distance from the wall.

$\delta$ : boundary layer thickness.

$L$ : distance of $U \times 1 \mathrm{~s}$ from the bubbles generated position. 